\title{
SYNTHESIS, SPECTRAL CHARACTERIZATION AND PHARMACOLOGICAL EVALUATION OF NOVEL THIAZOLE-OXOINDOLE HYBRID COMPOUNDS AS POTENTIAL ANTICANCER AGENTS
}

\author{
Yousry A. Ammar', A. M. Sh. El-Sharief ${ }^{1}$, Yehia A. Mohamed ${ }^{1}$, Ahmed B. M. Mehany ${ }^{2}$ and \\ Ahmed Ragab $^{1}$ \\ ${ }^{1}$ Chemistry Department, Faculty of Science, Al-Azhar University, Nasr City, Cairo, 11284, Egypt. \\ ${ }^{2}$ Zoology Department, Faculty of Science, Al-Azhar University, Nasr City, Cairo, 11284, Egypt.
}

\begin{abstract}
In the present study, a series of thiazole derivatives bearing 5-morpholinosulfonylindole were designed and synthesized through condensation of 5-(morpholinosulfonyl)isatin (1) with some thiazolidinone derivatives as carbon nucleophiles to afford the corresponding arylidene derivatives $(2-5,7,8)$. Furthermore, many thiazole derivatives (11-15) were obtained through the reaction of thiosemicarbazone derivative (10) with many reagents such as dimethyl acetelenedicarboxylate, phencylbromide derivatives, chloroacetonitrile, chloroacetylchloride as well as chloroacetanilide. The newly synthesized compounds were characterized based on spectral $\left(F T-I R,{ }^{l} H\right.$ $\left.N M R,{ }^{13} C N M R, M S\right)$ analysis. Cytotoxicity effects of all synthesized products were tested against three cancer cell lines, MCF-7, HepG-2, HCT-116 and they showed moderate to good cytotoxic activity against the three tested cell lines. The study showed that compounds $(2 a, 12 b$ and $12 \mathrm{c})$ were less or almost equipotent as doxorubicin against the three cell lines HepG-2, HCT-116.
\end{abstract}

Keywords: Isatin; thiazol-4(5H)-one; 4-thiazolidinone; thiosemicarbazone derivatives.

\section{1- INTRODUCTION}

Despite chemotherapy, surgery, radiotherapy, biotherapy and other adjuvant therapy are common treatments for tumor therapy [1] but chemotherapy is still the preferred and most significant treatment for cancer patients in the clinical application [2]. In the recent years there has been a surge of interest in heterocyclic compounds containing nitrogen and sulfur mainly because of their promising medicinal properties especially thiazole. Thiazoles are one of the most intensively investigated classes of aromatic five membered heterocycles and immense attention because it used as building block for the synthesis of many biologically active molecules as Sulfathiazole (antimicrobial drug), Nitazoxanide (antiprotozoal agent), Nizatidine (ulcer therapeutic), Abafungin (antifungal drug), Tiazofurin (antineoplastic agent), Meloxicam (non-steroidal, anti-inflammatory drug), epothilones and Antiarrhythmic, Anticoagulant activities [3-8] as shown in figure (1). Also, some natural products containing thiazole ring have been isolated as thiamine (vitamin B1), thiamine pyrophosphate (TPP, a coenzyme important in respiration in the Krebs cycle) $[9,10]$ and most of them exhibited significant cytotoxicity and antitumor potential [11-13]. Thus, the thiazole nucleus has been much studied in the field of organic and medicinal chemistry.

On the other hand, isatin is an endogenous compound identified in many organisms and possess diverse biological activities such as anti-bacterial [14], anticancer [15,16], anti-HIV [17], antimalarial [18], and anti TB activities [19], anti-oxidant [20] etc.

Also, sulfonamide derivatives display extensive biological properties, which are very popular among pharmaceutical chemists working on the design and synthesis of biologically active compounds in the pharmaceutical and agrochemical industry. This attractive scaffold displays a wide variety of pharmacological activities like anticancer [21], antibacterial [22] and anti-inflammatory [23] activities. 


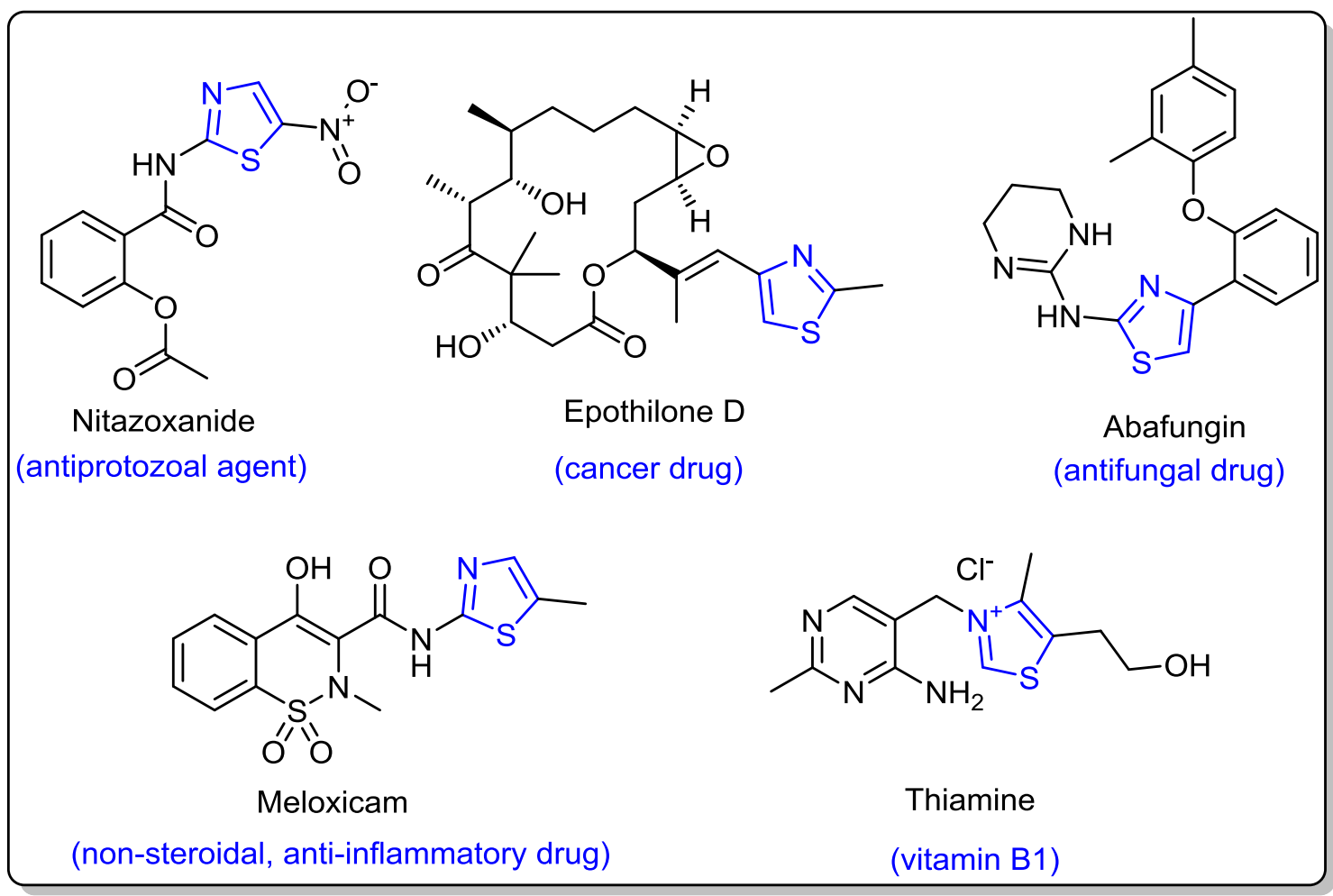

Fig. (1); Some reported drugs containing thiazoles.

In addition, the morpholine scaffold had found to be an outstanding pharmacophore in medicinal chemistry and several molecules having morpholine skeleton are the clinically approved drugs [24]. N-substituted morpholines used in the treatment of inflammatory diseases, like migraine and asthma [25]. Morpholine derivatives have been reported as platelet aggregation inhibitors, antiemetics, and bronchodilators [26]. Morpholine analogs establish a new antifungal chemical entity not allied with other presently available medications with antifungal potential [27].

Molecular hybridization is a valuable structure modification approach that comprises the incorporation of two or more pharmacophores into single entity. In view of the importance of thiazole and isatin derivatives and as part of our continued interest [28-31] in developing new active therapeutic agent (hybrid drug). We herein report the synthesis of novel thiazole based isatin sulphonamide.

\section{RESULTS AND DISCUSSION}

\subsection{Chemistry}

The synthetic pathway leading to the title compounds is outlined in Schemes 1-3. The key starting material 5-(morpholinosulfonyl) isatin (1) was prepared as previous described [28]. Thus, reaction of (1) with 3-alkyl-2thioxothiazolidin-4-one as carbon nucleophile in the presence of acetic acid containing fused sodium acetate furnished 5-(5(morpholinosulfonyl)-2-oxoindolin-3-ylidene)3-alkyl-2-thioxo- thiazolidin-4-one (2a,b). The structures of compounds $\mathbf{2 a}, \mathbf{b}$ were established on the basic of elemental analysis and spectral data. For example, the IR spectra of compounds 2a revealed absorption bands at $v 3160$ and $1696 \mathrm{~cm}^{-1}$ characterized for $\mathrm{NH}$ and $\mathrm{C}=\mathrm{O}$ groups, respectively. Its ${ }^{1} \mathrm{H}-\mathrm{NMR}$ spectra (DMSO- $d_{6}$ ) showed, in addition to the aromatic protons between 7.40-7.60 ppm related to eight protons, two triplet signals and one singlet signals at $\delta$ 2.84, 3.61 and $11.77 \mathrm{ppm}$ assignable to two $-\mathrm{CH}_{2}$ of morpholinyl moiety and one $\mathrm{NH}$ protons, respectively. 
Furthermore, the reaction of 5(morpholinosulfonyl)isatin (1) with 2iminothiazolidin-4-one afforded the corresponding 2-imino-5-(5-(morpholinosulfonyl)-2-oxoindolin-3-ylidene) thiazolidin4-one (3). The structure was assigned based on analytical and spectral data. IR spectra of compounds 3 , displayed absorption bands for $\mathrm{NH}, \mathrm{NH}_{2}$ at $v 3510,3222$ and $3114 \mathrm{~cm}^{-1}$ in addition to absorption bands at 1699 and 1612 $\mathrm{cm}^{-1}$ characterizing $\mathrm{C}=\mathrm{O}$ and $\mathrm{C}=\mathrm{N}$ functional groups, respectively. ${ }^{1} \mathrm{H}$ NMR spectra (DMSO$d_{6}$ ) of compounds $\mathbf{3}$ exhibited two triplet signals at $\delta 2.90$ and $3.66 \mathrm{ppm}$ indicating morpholinyl protons, three aromatic protons in region 7.17-9.43 ppm as well as two broad signals at $\delta 9.75,11.56 \mathrm{ppm}$ related to $\mathrm{NH}_{2}, \mathrm{NH}$ groups.

On the same manner, 2-(arylamino)-5-(5(morpholinosulfonyl)-2-oxoindolin-3-ylidene)
thiazol-4(5H)-ones (4a-c) were obtained through the reaction of compound (1) with 2(arylimino) thiazolidin-4-ones. Structure of the latter products was elucidated based on analytical and spectral data. IR spectrum of $\mathbf{4 a}$ revealed absorption bands at $v 3163,3129$ and $1699,1650 \mathrm{~cm}^{-1}$ corresponding to two $\mathrm{NH}$ and $2 \mathrm{C}=\mathrm{O}$ functional groups, respectively.

${ }^{1} \mathrm{H}$ NMR spectrum (DMSO- $d_{6}$ ) exhibited two singlet signals at $\delta 9.42$ and $11.61 \mathrm{ppm}$ assignable to two $\mathrm{NH}$, in addition to a multiplet signal at $\delta$ 7.10-7.81 ppm distinctive for aromatic protons. In addition, ${ }^{13} \mathrm{C}$ NMR spectrum of $\mathbf{4 c}$ displayed signals at $\delta 46.34$ and $65.67 \mathrm{ppm}$ for $\mathrm{CH}_{2}$-morpholinyl, also two signals were observed at 154.17 and 168.89 ppm corresponding to the two carbonyl groups.

Also, treatment of (1) with 2-(4-oxo-2thioxothiazolidin-3-yl) acetic acid produces 2-

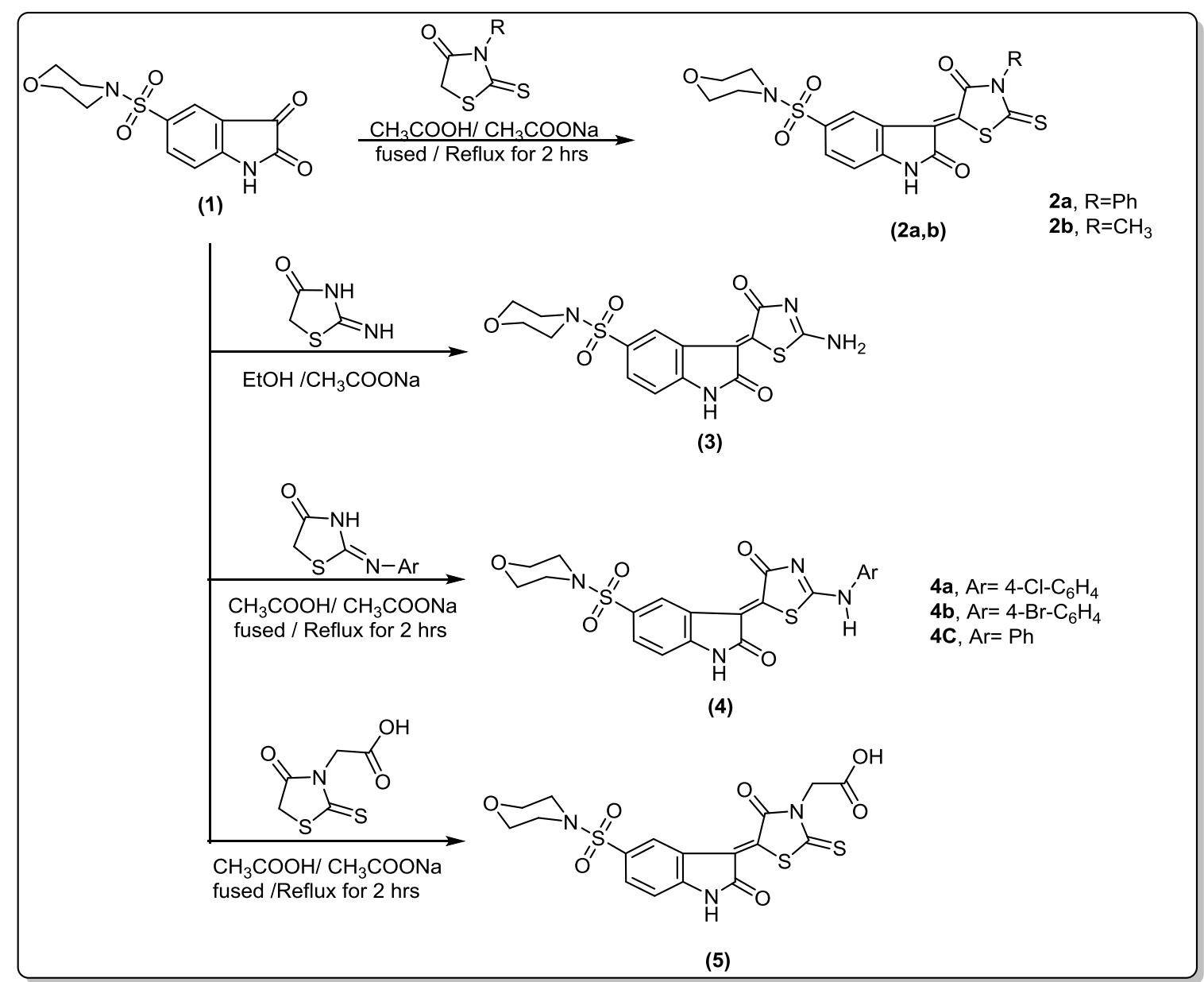

Scheme (1); Reaction of starting material 5-(morpholinosulfonyl)isatin (1) with different thiazole derivatives as carbon nucleophiles. 
(5-(5-(morpholinosulfonyl)-2-oxoindolin-3ylidene)-4-oxo-2-thioxothiazolidin-3-yl) acetic acid (5) based on elemental analysis and spectral data. The IR spectrum of that product revealed intense absorption broad band at $v$ 3453 for hydroxyl and NH groups and 1705, $1613 \mathrm{~cm}^{-1}$ related to carbonyl and $\mathrm{C}=\mathrm{N}$ groups. ${ }^{1} \mathrm{H}$ NMR spectra (DMSO- $d_{6}$ ) of compounds (5) exhibited two triplet signals at $\delta 2.92$ and 3.66 ppm indicating morpholinyl protons, $4.65 \mathrm{ppm}$ due to $\mathrm{CH}_{2}$ of acetic acid, three aromatic proton in region 7.22-9.22 ppm as well as $11.85 \mathrm{ppm}$ related to NH; Scheme (1).

2-(4-Oxothiazolidine-2-ylidene)acetonitrile (6) [32] characterized by the presence of two active methylene as well as presence of nitrile and carbonyl groups which make it chemically very active, so it can be used as a precursor to synthesize many biologically and chemically active ring systems. So, regioselective condensation was occurred through the reaction of acetonitrile derivatives (6) with the starting material (1) in acetic acid containing catalytic amount of fused sod. acetate, where the condensation occurred at the methylene group number 5 to afford a single product which was formulated as 2-(5-(5-(morpholinosulfonyl)-2oxoindolin-3-ylidene)-4-oxo-4,5-

dihydrothiazol-2-yl)acetonitrile (7). The structure of (7) was confirmed on the basis of analytical and spectroscopic data. Thus, IR spectrum showed stretching significance absorption bands at $v 3336,3219,2205$, and $1703 \mathrm{~cm}^{-1}$ attributed to two imine, nitrile, and carbonyl moieties. ${ }^{1} \mathrm{H}$ NMR spectra (DMSO$d_{6}$ ) of compounds (7) exhibited two triplet signals at $\delta 2.89$ and $3.64 \mathrm{ppm}$ indicating morpholinyl protons, $5.12 \mathrm{ppm}$ due to at exocyclic methylene of acetonitrile derivative (6), three aromatic proton in reign 7.10- 7.62 ppm as well as 9.6, $11.34 \mathrm{ppm}$ related to two $\mathrm{NH}$, This, prove that, 5-(morpholinosulfonyl) isatin condensed first with 4-thiazolidinone ring (6) at endocyclic methylene at position 5 rather than the most active methylene exocyclic methylene at position number 2 . Scheme 2 .

On the other hand, compound 2-(5(morpholinosulfonyl)-2-oxoindolin-3-ylidene)2-(4-oxo-4, 5-dihydrothiazol-2-yl) acetonitrile (8) has been synthesized through another route

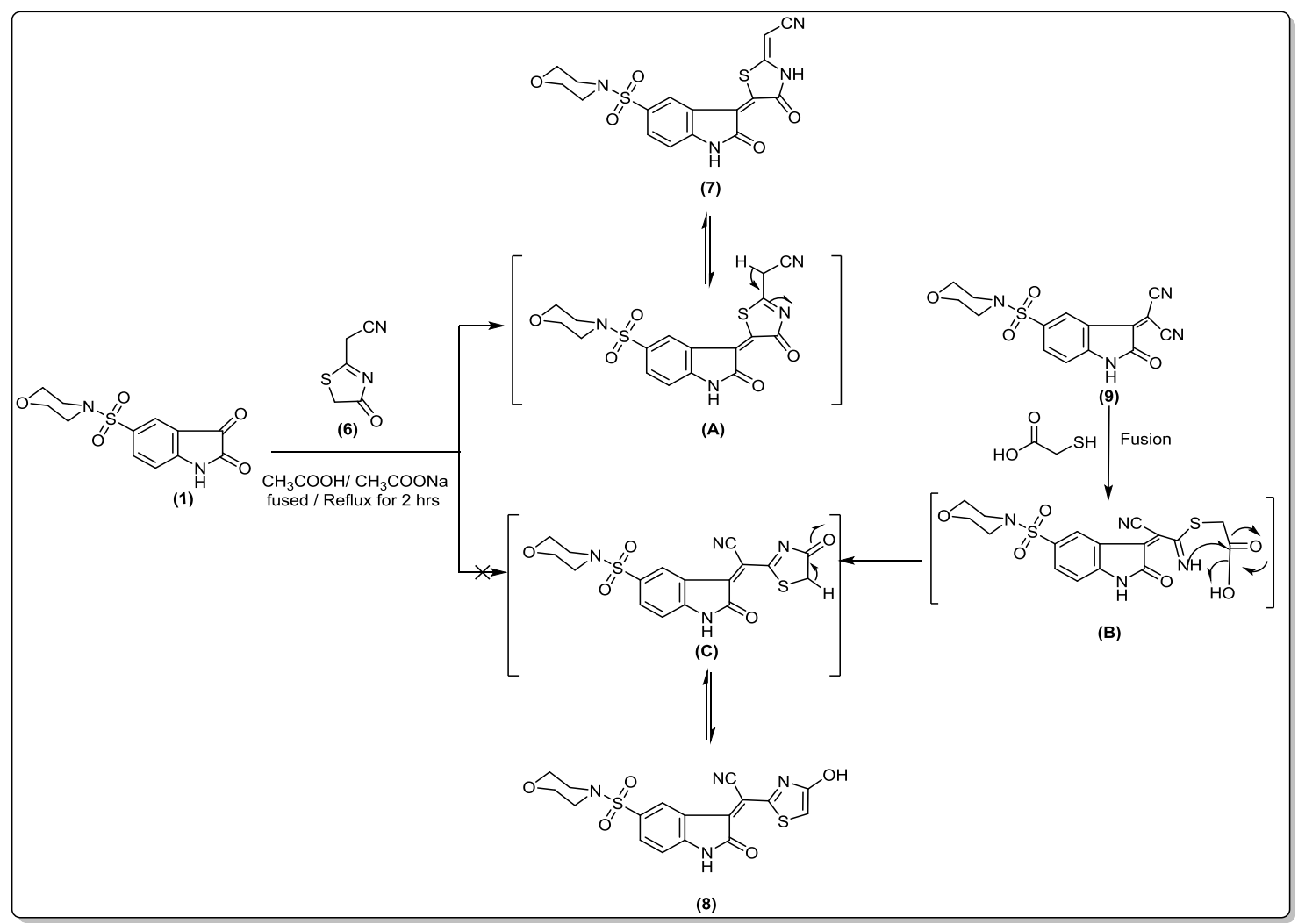

Scheme (2); Regioselective condensation of acetonitrile derivatives (6) with the starting material (1). 
via the reaction of 2-(5-(morpholinosulfonyl)2-oxoindolin-3-ylidene) malononitrile [28] with thioglycolic acid and the structure of the latter product was confirmed by spectroscopic studies and elemental analysis. The IR spectrum of compound (8) revealed absorption bands at $v$ $3349,3217,2207$ and $1740 \mathrm{~cm}^{-1}$ corresponding to an $\mathrm{OH}, \mathrm{NH}$, cyano and carbonyl groups. Its ${ }^{1} \mathrm{H}$ NMR spectrum (DMSO- $d_{6}$ ) revealed signals at $\delta 8.65$ and $11.56,12.08 \mathrm{ppm}$ due to methine$\mathrm{H}, \mathrm{NH}$ and $\mathrm{OH}$ protons where $\mathrm{NH}, \mathrm{OH}$ signals exchangeable by $\mathrm{D}_{2} \mathrm{O}$.

This investigation was extended to cover the reactivity of isatin thiosemicarbazone (10) [33] towards different reagents for the synthesis of many derivatives of thiazole containing indole moiety. Thus, the thiosemicarbazone derivative have tautomeric structure between thione / was discussed react as thiol derivative with the reagent as mentioned in scheme (3).
Interaction of compound (10) with dimethylacetelenedicarboxylate in methanol afforded one product which was identified as methyl 2-(2-(2-(-5-(morpholinosulfonyl)-2oxoindolin-3-ylidene)hydrazinyl)-4-oxothiazol5-(4H)-ylidene)acetate(11). The structure of (11) was eluci-dated by correct spectral data and elemental analyses. Its IR spectrum displayed stretching bands for $\mathrm{NH}$ and $2 \mathrm{CO}$ at $v$ 3190, $1732 \& 1701 \mathrm{~cm}^{-1}$. The ${ }^{1} \mathrm{H}$ NMR spectrum (DMSO- $d_{6}$ ) exhibited two singlet signals at $\delta 3.81,6.77$ due to methoxy and -CH methine exocyclic as well as two singlet signals due to two $\mathrm{NH}$ at $11.27,11.64 \mathrm{ppm}$ in addition to the morpholinyl and aromatic protons. Also, ${ }^{13} \mathrm{C}$-NMR spectrum showed presence of morpholinyl, methoxy, aromatic and methine carbons at 46.21, 46.32, 65.71 and 65.72, $53.11\left(\mathrm{OCH}_{3}\right), 111.60-158.88 \mathrm{ppm}$ in addition to three singlet signals at $\delta 163.16,166.35$, 168.98 related to carbonyl groups.

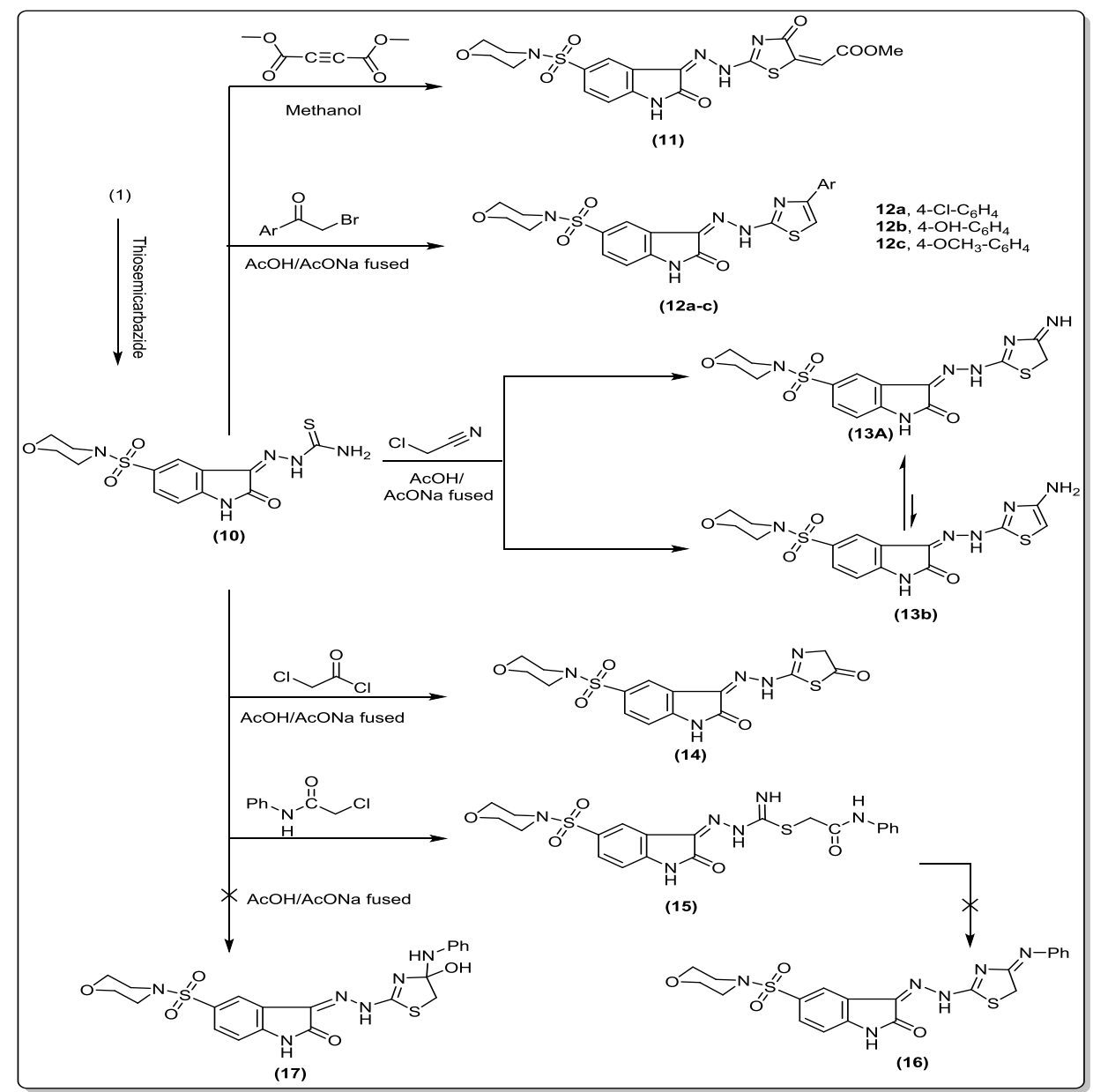

Scheme (3); Reaction of thiosemicarbazone with different reagents to afford the thiazole derivatives (11-15). 
In addition, 3-(2-(4-arylthiazol-2-yl) hydrazono)-5-(morpholinosulfonyl)indolin-2ones (12a-c) were produced through alkylation of the thiosemicarbazone derivative (10) with phencylbromides in acetic acid containing sod.acetate. IR pattern of (12a-c) displayed stretching frequencies at $v$ 3418-3104 and $1698-1691 \mathrm{~cm}^{-1}$ for $\mathrm{NH}, \mathrm{C}=\mathrm{O}$ respectivelly. ${ }^{1} \mathrm{H}$ NMR spectra (DMSO- $d_{6}$ ) of compound (12c) exhibited two triplet signals at $\delta 2.90$ and 3.65 ppm indicating morpholinyl protons, $3.89 \mathrm{ppm}$ due to methoxy group, seven aromatic protons in reign 7.06- $8.10 \mathrm{ppm},-\mathrm{CH}$ thiazole at $\delta 8.58$ ppm and two singlet signals at $\delta 10.97,11.73$ ppm related to NH. ${ }^{13} \mathrm{C}$ NMR spectrum of $\mathbf{1 2 b}$ displayed signals at $\delta 46.38,46.43,65.71$ and

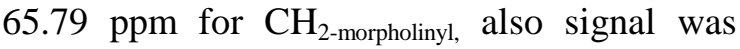
observed at $172.53 \mathrm{ppm}$ corresponding to carbonyl groups and aromatic carbon between 112.00- 163.16.

Treatment of thiosemicarbazone derivatives (10) with chloroacetonitrile in acetic acid afforded 3-(2-(4-imino-4,5-dihydrothiazol-2-yl) hydrazono)-5-(morpholino-sulfonyl) indolin-2one (13a) which was found to be in tautomeric forms with the isomer (13b), while isomer 13a was favored on the basis of spectral data. The IR spectrum of compound (13a) revealed absorption bands in the zone $v 3288,3196$ and $1700 \mathrm{~cm}^{-1}$ corresponding to an imine and carbonyl, respectively. Its ${ }^{1} \mathrm{H}$ NMR spectrum (DMSO- $d_{6}$ ) showed presence of $\mathrm{CH}_{2}$ of thiazole at 4.02 and lack of amino group and $\mathrm{CH}$-methine signals, also, three singlet signals were appeared at 8.52, 11.22, $11.65 \mathrm{ppm}$ corresponding for three $\mathrm{NH}$ proton which disappeared with $\mathrm{D}_{2} \mathrm{O}$.

In the same way 2-(2-(5(Morpholinosulfonyl)-2-oxo-indolin-3-

ylidene)hydrazinyl)thiazol-5(4H)-one (14) was obtained as a single product, when compound (10) was treated with chloroacetylchloride. Elemental analysis and spectral data were found in good agreement with the expected structure. IR spectrum revealed the absorption bands at $v 3284,3194$ related to $2 \mathrm{NH}$, in addition to $v 1700$ and $1613 \mathrm{~cm}^{-1}$ corresponding to $-\mathrm{C}=\mathrm{O}$ and $-\mathrm{C}=\mathrm{N}$ groups respectively. The ${ }^{1} \mathrm{H}$ NMR spectrum (DMSO- $d_{6}$ ) showed singlet signal at $\delta 4.04 \mathrm{ppm}$ attributed to methylene group, two triplets at $2.88,3.65 \mathrm{ppm}$ related to morpholinyl moiety and two singlet signals at $11.23,11.65 \mathrm{ppm}$ for $-\mathrm{NH}$ protons respectively, beside signals at $\delta 7.17-8.10 \mathrm{ppm}$, which are distinctive for aromatic protons, that identified as two doubles and one singlet for indolinone moiety.

On another hand, interaction of compound (10) with chloroacetanilide derivative produced a product which was formulated as the noncyclic structure $\mathbf{1 5}$ rather than the cyclic structure $\mathbf{1 6}$ or $\mathbf{1 7}$. The structure of (15) was confirmed based on the elemental analysis and spectral data. Thus, its IR spectrum showed the presence of many bands at $v$ 3283, 3182 and $1725 \mathrm{~cm}^{-1}$ corresponding for $\mathrm{NH}$ and $\mathrm{C}=\mathrm{O}$. Its ${ }^{1} \mathrm{H}$ NMR spectrum exhibited two triplet signals at $\delta 2.89(J=4.0 \mathrm{~Hz})$ and $3.65(J=4.2 \mathrm{~Hz})$, singlet signal at $\delta 4.04$ corresponding for the methylene group beside four singlet signals at $\delta$ $10.03,11.23,11.65,12.87 \mathrm{ppm}$ due to $4 \mathrm{NH}$ as well as eight aromatic protons between 7.08$8.52 \mathrm{ppm} .{ }^{13} \mathrm{C}$ NMR spectrum of (15) displayed signals at $\delta 21.51$ for methylene group, 46.37, 46.42, 65.72 and $65.79 \mathrm{ppm}$ related to $\mathrm{CH}_{2}$ of morpholinyl moiety, also three signals were observed at 174.8, 175.85, $179.26 \mathrm{ppm}$ corresponding to $\mathrm{C}=\mathrm{N}$ and carbonyl groups beside signals of aromatic proton in the range 111.2-165.30 ppm, also mass spectrum showed a molecular ion peak at $\mathrm{m} / \mathrm{z} 502$ corresponding to the molecular formula.

\subsection{Biological evaluation}

The target compounds were evaluated for the cytotoxicity against three cancer cell lines including HepG-2, HCT-116 and MCF-7 using sulforhodamine B colorimetric (SRB) cell proliferation assay, doxorubicin was used as a reference, the obtained $\mathrm{IC}_{50}$ values are expressed in $\mu \mathrm{M}$ and represented in Table 1.

The target compounds showed moderate to good cytotoxic activity against the three tested cell lines where most of the tested compounds 
have shown broad spectrum towards the selected cell lines HepG-2, HCT-116 and MCF-7 cell line.

Compounds 2a, 12b and 12c exhibited the best activity against HepG-2, HCT-116 and MCF-7 with $\mathrm{IC}_{50}$ values of $(6.59 \pm 2.14,8.78 \pm$ $2.5,7.76 \pm 0.18) \mu \mathrm{M}$ for compound $\mathbf{2 a}, \quad(4.37$ $\pm 0.22,6.86 \pm 0.14,9.77 \pm 0.25) \mu \mathrm{M}$ for compound $\mathbf{1 2 b}$ and $(9.44 \pm 1.65,7.83 \pm 0.58$, $5.49 \pm 0.68) \mu \mathrm{M}$ for compound $\mathbf{1 2 c}$ which were less active or equipotent as doxorubicin.

Table 1: Cytotoxic activity of the synthesized compounds against cancerous cell lines.

\begin{tabular}{|c|c|c|c|}
\hline \multirow{2}{*}{ Sample no. } & \multicolumn{3}{|c|}{ IC $_{50} \%(\mu \mathrm{M}) \pm$ S.E. } \\
\cline { 2 - 4 } & HepG-2 & HCT-116 & MCF-7 \\
\hline 1 & $28.54 \pm 2.5$ & $59.97 \pm 0.09$ & $67.93 \pm 0.19$ \\
\hline $2 a$ & $6.59 \pm 2.14$ & $8.78 \pm 2.5$ & $7.76 \pm 0.18$ \\
\hline $2 b$ & $18.49 \pm 1.28$ & $13.53 \pm 0.35$ & $20.61 \pm 1.12$ \\
\hline 3 & $35.95 \pm 2.60$ & $40.51 \pm 3.8$ & $34.36 \pm 0.85$ \\
\hline $4 a$ & $23.86 \pm 0.54$ & $23.82 \pm 1.35$ & $28.80 \pm .68$ \\
\hline $4 b$ & $40.09 \pm 1.68$ & $33.46 \pm 0.81$ & $42.09 \pm 2.21$ \\
\hline $4 c$ & $47.85 \pm 1.35$ & $44.63 \pm 0.45$ & $51.59 \pm 1.65$ \\
\hline 5 & $37.20 \pm 0.67$ & $41.27 \pm 0.87$ & $32.75 \pm 1.02$ \\
\hline 7 & $32.87 \pm 1.02$ & $36.69 \pm 0.28$ & $44.23 \pm 2.42$ \\
\hline 8 & $41.43 \pm 3.86$ & $39.18 \pm 2.48$ & $43.28 \pm 0.69$ \\
\hline 10 & $18.48 \pm 0.34$ & $15.51 \pm 0.25$ & $18.67 \pm 0.65$ \\
\hline 11 & $20.61 \pm 0.68$ & $23.44 \pm 2.81$ & $25.34 \pm 1.65$ \\
\hline $12 a$ & $43.33 \pm 1.65$ & $26.64 \pm 0.31$ & $46.32 \pm 2.54$ \\
\hline $12 b$ & $4.37 \pm 0.22$ & $6.86 \pm 0.14$ & $9.77 \pm 0.25$ \\
\hline $12 c$ & $9.44 \pm 1.65$ & $7.83 \pm 0.58$ & $5.49 \pm 0.68$ \\
\hline 13 & $20.49 \pm 1.87$ & $25.07 \pm 1.68$ & $30.27 \pm 0.98$ \\
\hline 14 & $31.24 \pm 0.58$ & $33.17 \pm 2.35$ & $37.45 \pm 0.71$ \\
\hline 15 & $15.72 \pm 1.92$ & $17.94 \pm 0.57$ & $20.86 \pm 2.21$ \\
\hline Isatin & $32.31 \pm 0.45$ & $21.56 \pm 0.37$ & $41.83 \pm 0.67$ \\
\hline Dox. & $5.59 \pm 0.55$ & $7.03 \pm 0.21$ & $4.89 \pm 0.47$ \\
\hline
\end{tabular}

**Three independent experiments were performed for each concentration.

The results indicated that the 3-alkyl-2thioxo- thiazolidin-4-one (2a, b) derivatives were more active than the corresponding 2iminothiazolidin-4-one derivatives (3), 2(arylamino)-5-(5-(morpholinosulfonyl)-2oxoindolin-3-ylidene) thiazol-4(5H)-ones (4ac), This can be attributed to presence of new thioxo in designed compounds in position 2 (2a,b) derivatives also, presence of (-N-Ph) more active than (-N-Me), see Table 1

Presence of acetic acid derivatives as 2-(5(5-(morpholinosulfonyl)-2-oxoindolin-3-

ylidene)-4-oxo-2-thioxothiazolidin-3-yl) acetic acid (5) as well as acetonitrile derivatives $(\mathbf{7 , 8})$ showed moderate anticancer activity, with $\mathrm{IC}_{50}$ value less than $50 \mu \mathrm{M}$ against all the tested cell line $(37.20 \pm 0.67,41.27 \pm 0.87,32.75 \pm 1.02)$ $\mu \mathrm{M}$ for $(\mathbf{5}),(32.87 \pm 1.02,36.69 \pm 0.28,44.23$ $\pm 2.42) \mu \mathrm{M}$ for $(7)$ and $(41.43 \pm 3.86,39.18 \pm$ $2.48,43.28 \pm 0.69) \mu \mathrm{M}$ for (8) respectively and from previous result it's clear that condensation at endocyclic methylene at position $\mathbf{5}$ at compound (7) give slightly significant against all cell lines rather than exocyclic methylene at position number 2 compound (8).

As found in table 1, it is readily observed that there is a significant difference in cytotoxic activity when various alkyl groups are introduced into the phenyl ring of phencylbromide derivatives in (12a-c). The Presence of hydroxy group in 4-arylthiazol-2-yl derivatives (12b) affected activity higher than doxorubicin against HepG-2 and HCT-116cell line, so replacement of hydroxyl group is required to study structure activity relationship. Introducing methoxy group to aryl group of 3(2-(4-(4-aryl) thiazol-2-yl) hydrazono)-5(morpholinosulfonyl) indolin-2-one (12a, c) causes an increase in activity in the cause of 12c rather than 12a, which may be attributed to a lipophilic electron withdrawing ( $\mathrm{Cl}$ atom).

Compounds $(13,14,15)$ showed moderate anticancer activity, against all the tested cell line and in some causes give result activity better than isatin itself and starting material and that indicate that sulfonyl moiety affected in activity and presence of thiazole derivative in the desired compounds increase activity against all the tested cell lines. Most of synthetized compounds showed good activity than starting material. Finally, the presence of 2-thioxo (2a, b) and presence of hydroxy group on 4-position of phenyl ring at 4-arylthiazol-2-yl derivatives (12b) enhanced the antitumor activity and led 
to results near to or better than the reference drug.

\section{EXPERIMENTAL SECTION}

\subsection{Chemistry}

All melting points are recorded on digital Gallen Kamp MFB-595 instrument and may be uncorrected. The IR spectra $(\mathrm{KBr})\left(\mathrm{cm}^{-1}\right)$ were measured on a Shimadzu 440 spectrophotometer. ${ }^{1} \mathrm{H}$ NMR spectra $(\delta, \mathrm{ppm})$ were obtained in deuterated dimethyl sulfoxide on a Bruker spectrometer (400 $\mathrm{MHz})$ spectrometer, using TMS as an internal standard; chemical shifts are reported as $\delta \mathrm{ppm}$ units. Mass spectra were recorded on Thermo Scientific ISQLT mass spectrometer at the Regional Center for Mycology and Biotechnology, Al-Azhar University. Elemental analyses were carried out at Micro Analytical Unit, Cairo University, Cairo, Egypt.

Synthesis of thiazole derivatives $2 a, b, 3$, 4,5,7 :-

A mixture of 5-morpholinosulfonylisatin (1) $(0.01 \mathrm{~mol})$ and 3-phenyl-2thioxothiazolidin-4-one, 3-methyl-2thioxothiazolidin-4-one, 2-iminothiazolidin-4one, 2-(arylamino)thiazol-4(5H)-one derivatives, 2-(4-oxo-4,5-dihydrothiazol-2yl)acetonitrile and/ or 2-(4-oxo-2thioxothiazolidin-3-yl)-acetic acid $(0.01 \mathrm{~mol})$ in $10 \mathrm{~mL}$ acetic acid containing $(0.2 \mathrm{~g})$ of fused sodium acetate was heated under reflux for 1-4 h. The solid product formed collected by filtration and washed by water and recrystallized from proper solvent.

\section{5-(5-(morpholinosulfonyl)-2-oxoindolin-3- ylidene)-3-phenyl-2-thioxothiazolidin-4-one (2a):-}

Yield $89.3 \%$ as red powder from ethanol; mp: $286-288^{\circ} \mathrm{C}$, IR: $v / \mathrm{cm}^{-1}: 3160(\mathrm{NH}), 3060$ (CH-Ar), 2974, 2911, 2848 (CH-aliph), 1696 $(\mathrm{C}=\mathrm{O}), 1617(\mathrm{C}=\mathrm{N}) ;{ }^{1} \mathrm{H}$ NMR: $\delta / \mathrm{ppm} 2.84(\mathrm{t}$, $\left.4 \mathrm{H}, J=4.2 \mathrm{~Hz}, \mathrm{~N}\left(\mathrm{CH}_{2}\right)_{2}\right), 3.61(\mathrm{t}, 4 \mathrm{H}, J=3.8 \mathrm{~Hz}$ $\left.\mathrm{O}\left(\mathrm{CH}_{2}\right)_{2}\right), 7.40-7.60(\mathrm{~m}, 8 \mathrm{H}, \mathrm{Ar}-\mathrm{H}), 11.77$ (s, $\mathrm{H}, \mathrm{NH}$; exchangeable with $\mathrm{D}_{2} \mathrm{O}$ ); MS: (Mwt.: 487): $\mathrm{m} / \mathrm{z}, 487\left[\mathrm{M}^{+1},(34.12 \%)\right], 56$ (100\%);
Anal. Calcd for $\mathrm{C}_{21} \mathrm{H}_{17} \mathrm{~N}_{3} \mathrm{O}_{5} \mathrm{~S}_{3}$ (487.56): C, 51.73; H, 3.51; N, 8.62; Found C, 51.81; H, $3.43 ; \mathrm{N}, 8.78 \%$.

\section{3-methyl-5-(5-(morpholinosulfonyl)-2- oxoindolin-3-ylidene)-2-thioxothiazolidin-4- one(2b):-}

Yield $92.8 \%$ as red powder from methanol; $\mathrm{mp}:>300^{\circ} \mathrm{C}$, IR: $v / \mathrm{cm}^{-1}: 3201(\mathrm{NH}), 3075$ (CH-Ar), 2975, 2895, 2856 (CH-aliph), 1718, $1693(\mathrm{C}=\mathrm{O}), 1613(\mathrm{C}=\mathrm{N}) ;{ }^{1} \mathrm{H}$ NMR: $\delta / \mathrm{ppm}=$ 2.92 (t, $\left.4 \mathrm{H}, J=4.0 \mathrm{~Hz}, \mathrm{~N}\left(\mathrm{CH}_{2}\right)_{2}\right), 3.48(\mathrm{~s}, 3 \mathrm{H},-$ $\left.\mathrm{N}-\mathrm{CH}_{3}\right), 3.67$ (t, $\left.4 \mathrm{H}, J=4.2 \mathrm{~Hz},-\mathrm{O}\left(\mathrm{CH}_{2}\right)_{2}\right), 7.22$ $(\mathrm{d}, 1 \mathrm{H}, J=8.4 \mathrm{~Hz}, \mathrm{Ar}-\mathrm{H}), 7.81(\mathrm{~d}, 1 \mathrm{H}, J=8.4$ $\mathrm{Hz}, \mathrm{Ar}-\mathrm{H}$ ), 9.26 (s, 1H, Ar-H), 11.78 (s, H, NH; exchangeable with $\mathrm{D}_{2} \mathrm{O}$ ); MS:(Mwt.: 425): $\mathrm{m} / \mathrm{z}$, $425\left[\mathrm{M}^{+1},(11.28 \%)\right], 56(100 \%) ;$ Anal. Calcd for $\mathrm{C}_{16} \mathrm{H}_{15} \mathrm{~N}_{3} \mathrm{O}_{5} \mathrm{~S}_{3}$ (425.49): C, 45.17; H, 3.55; N, 9.88; Found C, 45.32; H, 3.31; N, 9.94\%.

\section{2-imino-5-(5-(morpholinosulfonyl)-2- oxoindolin-3-ylidene) thiazolidin-4-one (3):-}

Yield $81.3 \%$ as yellowish powder from ethanol; mp: $290-292{ }^{\circ} \mathrm{C}$, IR: $v / \mathrm{cm}^{-1}$ : 3510, 3222, $3114\left(\mathrm{NH}, \mathrm{NH}_{2}\right), 3052$ (CH-Ar), 2968, 2905, 2859 (CH-aliph), 1699 (C=O), 1612 $(\mathrm{C}=\mathrm{N}) ;{ }^{1} \mathrm{H}$ NMR: $\delta / \mathrm{ppm} 2.90(\mathrm{t}, 4 \mathrm{H}, J=4.2$ $\left.\mathrm{Hz}, \mathrm{N}\left(\mathrm{CH}_{2}\right)_{2}\right), 3.66$ (t, $\left.4 \mathrm{H}, J=4.4 \mathrm{~Hz} \mathrm{O}\left(\mathrm{CH}_{2}\right)_{2}\right)$, $7.17(\mathrm{~d}, 1 \mathrm{H}, J=8.4 \mathrm{~Hz}, \operatorname{Ar}-\mathrm{H}), 7.73(\mathrm{~d}, 1 \mathrm{H}, J=$ $8.4 \mathrm{~Hz}, \mathrm{Ar}-\mathrm{H}), 9.43$ (s, 1H, Ar-H), 9.75(br, 2H, $\mathrm{NH}_{2}$ : exchangeable with $\mathrm{D}_{2} \mathrm{O}$ ), 11.56 (br, $1 \mathrm{H}$, $\mathrm{NH}$; exchangeable with $\mathrm{D}_{2} \mathrm{O}$ ); MS:(Mwt.: 394): $\mathrm{m} / \mathrm{z}, 394\left[\mathrm{M}^{+1},(10.13 \%)\right], 152$ (100\%); Anal. Calcd for $\mathrm{C}_{15} \mathrm{H}_{14} \mathrm{~N}_{4} \mathrm{O}_{5} \mathrm{~S}_{2}$ (394.42): $\mathrm{C}, 45.68 ; \mathrm{H}$, 3.58; N, 14.21; Found C, 45.62; H, 3.44; N, $14.35 \%$.

\section{2-((4-Chlorophenyl)amino)-5-(5- (morpholinosulfonyl)-2-oxoindolin-3- ylidene)thiazol-4(5H)-one) (4a) :-}

Yield $86.87 \%$ as reddish powder from ethanol; mp: $>300^{\circ} \mathrm{C}$, IR: $v / \mathrm{cm}^{-1}$ : 3166, 3133 (2NH), 3060 (CH-Ar), 2978, 2918, 2859 (CHaliph), 1699, $1650(\mathrm{C}=\mathrm{O}), 1613(\mathrm{C}=\mathrm{N}) ;{ }^{1} \mathrm{H}$ NMR: $\delta / p p m ~ 2.91\left(t, 4 \mathrm{H}, \mathrm{J}=4.0 \mathrm{~Hz}, \mathrm{~N}\left(\mathrm{CH}_{2}\right)_{2}\right)$, $3.66\left(\mathrm{t}, 4 \mathrm{H}, \mathrm{J}=4.2 \mathrm{~Hz} \mathrm{O}\left(\mathrm{CH}_{2}\right)_{2}\right), 7.1(\mathrm{~d}, 1 \mathrm{H}, J=$ $8.4 \mathrm{~Hz}, \mathrm{Ar}-\mathrm{H}), 7.15$ (d, 1H, J= 8.4 Hz, Ar-H), $7.18(\mathrm{~d}, 1 \mathrm{H}, J=8.0 \mathrm{~Hz}$, Ar-H), 7.47-7.49 (m, 2H, Ar-H), 7.51 (d, 1H, J= 8.4 Hz, Ar-H), 7.81 
(d, $1 \mathrm{H}, J=8.4 \mathrm{~Hz}, \mathrm{Ar}-\mathrm{H}), 9.42,11.61(2 \mathrm{~s}, 2 \mathrm{H}$, 2NH; exchangeable with $\left.\mathrm{D}_{2} \mathrm{O}\right)$; Anal. Calcd for $\mathrm{C}_{21} \mathrm{H}_{17} \mathrm{ClN}_{4} \mathrm{O}_{5} \mathrm{~S}_{2}$ (504.97): C, 49.95; H, 3.39; N, 11.10; Found C, 49.78; H, 3.51; N, 11.03\%.

\section{2-((4-Bromophenyl)amino)-5-(5-}

(morpholinosulfonyl)-2-oxoindolin-3ylidene)thiazol4 $(5 H)$-one( $4 \mathrm{~b})$ :-

Yield $88.54 \%$ as red powder from ethanol; mp: 291-293 ${ }^{\circ} \mathrm{C}$, IR: $v / \mathrm{cm}^{-1}: \quad 3163,3129$ (2NH), 3060 (CH-Ar), 2977, 2918, 2857 (CHaliph), 1699, $1648(\mathrm{C}=\mathrm{O}), 1613(\mathrm{C}=\mathrm{N}) ;{ }^{1} \mathrm{H}$ NMR: $\delta / p p m ~ 2.95\left(\mathrm{t}, 4 \mathrm{H}, J=4.2 \mathrm{~Hz}, \mathrm{~N}\left(\mathrm{CH}_{2}\right)_{2}\right)$, $3.66\left(\mathrm{t}, 4 \mathrm{H}, J=3.8 \mathrm{~Hz} \mathrm{O}\left(\mathrm{CH}_{2}\right)_{2}\right), 7.04(\mathrm{~d}, 1 \mathrm{H}$, $J=8.0 \mathrm{~Hz}$, Ar-H), 7.13-7.19 (m, 2H, Ar-H), $7.62(\mathrm{~d}, 1 \mathrm{H}, J=8.4 \mathrm{~Hz}, \operatorname{Ar}-\mathrm{H}), 7.63-7.64(\mathrm{~m}$, 1H, Ar-H), 7.7-7.72 (m, 1H, Ar-H), $7.77(\mathrm{~d}$, $1 \mathrm{H}, J=8.4 \mathrm{~Hz}$, Ar-H), 9.41, $11.63(2 \mathrm{~s}, 2 \mathrm{H}$, 2NH; exchangeable with $\left.\mathrm{D}_{2} \mathrm{O}\right) ;{ }^{13} \mathrm{C}$ NMR: $46.37\left(\mathrm{~N}\left(\mathrm{CH}_{2}\right)_{2}\right), 65.80\left(\mathrm{O}\left(\mathrm{CH}_{2}\right)_{2}\right), 111.03$, $120.91, \quad 121.15, \quad 123.49, \quad 124.22, \quad 127.66$, $128.06, \quad 131.60, \quad 132.49, \quad 132.77, \quad 141.24$, 144.90, 146.62, 147.05, 147.09, 152.84, 169.54 $(2 \mathrm{C}=\mathrm{O})$; Anal. Calcd for $\mathrm{C}_{21} \mathrm{H}_{17} \mathrm{BrN}_{4} \mathrm{O}_{5} \mathrm{~S}_{2}$ (549.41): C, 45.91; H, 3.12; N, 10.20; Found C, 45.82; H, 3.27; N, $10.33 \%$.

\section{5-(5-(Morpholinosulfonyl)-2-oxoindolin-3-} ylidene)-2-(phenylamino)thiazol-4(5H)-one( 4c):-

Yield $84.34 \%$ as orange powder from ethanol; mp: $298-300{ }^{\circ} \mathrm{C}$, IR: $v / \mathrm{cm}^{-1}$ : 3162, $(\mathrm{NH}), 3058$ (CH-Ar), 2980, 2919, $2859(\mathrm{CH}-$ aliph), 1699, $1648(\mathrm{C}=\mathrm{O}), 1613(\mathrm{C}=\mathrm{N}) ;{ }^{1} \mathrm{H}$ NMR: $\delta / p p m ~ 2.93\left(\mathrm{t}, 4 \mathrm{H}, J=4.0 \mathrm{~Hz}, \mathrm{~N}\left(\mathrm{CH}_{2}\right)_{2}\right)$, 3.64(t, $\left.4 \mathrm{H}, J=4.2 \mathrm{~Hz} \mathrm{O}\left(\mathrm{CH}_{2}\right)_{2}\right), 7.07(\mathrm{~d}, 1 \mathrm{H}$, $J=8.4 \mathrm{~Hz}, \mathrm{Ar}-\mathrm{H}), 7.14(\mathrm{~d}, 1 \mathrm{H}, J=8.4 \mathrm{~Hz}, \mathrm{Ar}-$ $\mathrm{H})$, 7.33-7.35 (m, 2H, Ar-H), $7.53(\mathrm{~d}, 1 \mathrm{H}, J=$ 8.4 Hz, Ar-H), 7.66-7.69 (m, 2H, Ar-H), 7.73$7.75(\mathrm{~m}, 1 \mathrm{H}, \mathrm{Ar}-\mathrm{H}), 9.41,11.62(2 \mathrm{~s}, 2 \mathrm{H}, 2 \mathrm{NH}$; exchangeable with $\left.\mathrm{D}_{2} \mathrm{O}\right)$; ${ }^{13} \mathrm{C}$ NMR: 46.34 $\left(\mathrm{N}\left(\mathrm{CH}_{2}\right)_{2}\right), 65.67\left(\mathrm{O}\left(\mathrm{CH}_{2}\right)_{2}\right), 110.13,121.21$, $122.67,123.99,124.27,126.76,129.36$, $131.32, \quad 133.17, \quad 133.84,142.38,145.90$, $146.67, \quad 147.10, \quad 147.99, \quad 154.17, \quad 168.89$ $(2 \mathrm{C}=\mathrm{O}) ; \quad$ MS:(Mwt.:470): $\mathrm{m} / \mathrm{z}, \quad 470\left[\mathrm{M}^{+1}\right.$, (38.44\%)], 407 (100\%);Anal. Calcd for
$\mathrm{C}_{21} \mathrm{H}_{18} \mathrm{~N}_{4} \mathrm{O}_{5} \mathrm{~S}_{2}$ (470.52): C, 53.61; H, 3.86; N, 11.91; Found C, 53.46; H, 3.98; N, 11.84\%.

2-(5-(5-(Morpholinosulfonyl)-2-oxoindolin-3ylidene)-4-oxo-2-thioxothiazolidin-3-yl)acetic acid(5):-

Yield $92 \%$ as red powder from ethanol; mp: dec. $280{ }^{\circ} \mathrm{C}$, IR: $v / \mathrm{cm}^{-1}: 3453$ ( $\mathrm{br}$. OH,NH), 3069 (CH-Ar), 2969, 2902, 2862 (CH-aliph), $1705(\mathrm{C}=\mathrm{O}), 1613(\mathrm{C}=\mathrm{N}) ;{ }^{1} \mathrm{H}$ NMR: $\delta / p p m ~ 2.92$ (t, 4H, $\left.J=4.0 \mathrm{~Hz}, \mathrm{~N}\left(\mathrm{CH}_{2}\right)_{2}\right)$, $3.66\left(\mathrm{t}, 4 \mathrm{H}, J=4.0 \mathrm{~Hz}, \mathrm{O}\left(\mathrm{CH}_{2}\right)_{2}\right), 4.65(\mathrm{~s}, 2 \mathrm{H}$, $\left.\mathrm{CH}_{2}\right), 7.22(\mathrm{~d}, 1 \mathrm{H}, J=8.4 \mathrm{~Hz}, \mathrm{Ar}-\mathrm{H}), 7.81(\mathrm{~d}$, $1 \mathrm{H}, J=8.8 \mathrm{~Hz}, \mathrm{Ar}-\mathrm{H}), 9.22$ (s, 1H, Ar-H), 11.85 (s, $1 \mathrm{H}, \quad \mathrm{NH}$; exchangeable with $\mathrm{D}_{2} \mathrm{O}$ ); MS:(Mwt.: 469): m/z, 469[M $\left.{ }^{+1},(16.97 \%)\right], 134$ (100\%); Anal. Calcd for $\mathrm{C}_{17} \mathrm{H}_{15} \mathrm{~N}_{3} \mathrm{O}_{7} \mathrm{~S}_{3}$ (469.50): C, 43.49; H, 3.22; N, 8.95; Found C, $43.35 ; \mathrm{H}, 3.41 ; \mathrm{N}, 8.87 \%$.

2-(5-(5-(morpholinosulfonyl)-2-oxoindolin-3ylidene)-4-oxo-4,5-dihydrothiazol-2-yl) acetonitrile (7):-

Yield $68.14 \%$ as brown powder from ethanol/ benzene; mp: dec. $280{ }^{\circ} \mathrm{C}$, IR: $v / \mathrm{cm}^{-1}$ : 3336, 3219 (2NH), 3086 (CH-Ar), 2976, 2921, 2858 (CH-aliph), 2205 (CN), 1703(br-C=O), $1612(\mathrm{C}=\mathrm{N}) ;{ }^{1} \mathrm{H}$ NMR: $\delta / \mathrm{ppm}=2.89(\mathrm{t}, 4 \mathrm{H}$, $\left.\mathrm{N}\left(\mathrm{CH}_{2}\right)_{2}\right), 3.64$ (t, 4H, O $\left.\left(\mathrm{CH}_{2}\right)_{2}\right), 5.12$ (s, CHmethine), $7.1(\mathrm{~d}, 1 \mathrm{H}, \mathrm{J}=8.4 \mathrm{~Hz}, \mathrm{Ar}-\mathrm{H}), 7.12(\mathrm{~s}$, 1H, Ar-H), 7.62 (d, 1H, 8.0, Ar-H), 9.6, 11.43 $\left(2 \mathrm{~s}, 2 \mathrm{H}, 2 \mathrm{NH}\right.$; exchangeable with $\left.\mathrm{D}_{2} \mathrm{O}\right)$; Anal. Calcd for $\mathrm{C}_{17} \mathrm{H}_{14} \mathrm{~N}_{4} \mathrm{O}_{5} \mathrm{~S}_{2}$ (418.44): C, 48.80; $\mathrm{H}$, 3.37; N, 13.39; Found C, 48.65; H, 3.22; N, $13.26 \%$.

Synthesis of 2-(5-(morpholinosulfonyl)-2oxoindolin-3-ylidene)-2-(4-oxo-4,5-dihydrothiazol2-yl) acetonitrile (8):-

A mixture of 2-(5-(morpholinosulfonyl)-2oxoindolin-3-ylidene) malononitrile $(0.01 \mathrm{~mol})$ and thioglycolic acid $(0.01 \mathrm{~mol})$ were heated under reflux for $6 \mathrm{~h}$ in the presence of $(10 \mathrm{ml})$ of dimethylformamide. The mixture was concentrated and cooled to room temperature then poured on crushed ice, the separated solid was filtered, washed several times with water, 
dried and recrystallized to give the title compound.

Yield $71.68 \%$ as brown powder from dioxane ; mp: $240-242{ }^{\circ} \mathrm{C}$, IR: $v / \mathrm{cm}^{-1}$ : 3349, 3217 (OH, NH), 3067 (CH-Ar), 2979, 2921, 2860 (CH-aliph), $2207(\mathrm{CN}), 1740(\mathrm{C}=\mathrm{O}), 1616$ $(\mathrm{C}=\mathrm{N}) ;{ }^{1} \mathrm{H}$ NMR: $\delta / \mathrm{ppm} 2.91(\mathrm{t}, 4 \mathrm{H}, J=4.6 \mathrm{~Hz}$, $\left.\mathrm{N}\left(\mathrm{CH}_{2}\right)_{2}\right), 3.65\left(\mathrm{t}, 4 \mathrm{H}, J=2.8 \mathrm{~Hz}, \mathrm{O}\left(\mathrm{CH}_{2}\right)_{2}\right)$, $7.11(\mathrm{~d}, 1 \mathrm{H}, J=8.0 \mathrm{~Hz}, \mathrm{Ar}-\mathrm{H}), 7.67(\mathrm{~d}, 1 \mathrm{H}, J=$ 8.0, Ar-H), $8.08(\mathrm{~s}, 1 \mathrm{H}, \mathrm{Ar}-\mathrm{H}), 8.65$ (s, CHmethine), 11.56, 12.08 (2s, 2H, NH-isatin , OH; exchangeable with $\mathrm{D}_{2} \mathrm{O}$ ); Anal. Calcd for $\mathrm{C}_{17} \mathrm{H}_{14} \mathrm{~N}_{4} \mathrm{O}_{5} \mathrm{~S}_{2}$ (418.44): $\mathrm{C}, 48.80 ; \mathrm{H}, 3.37 ; \mathrm{N}$, 13.39; Found C, 48.72; H, 3.29; N, $13.41 \%$.

\section{Synthesis of methyl-2-(2-(2-(-5- (morpholinosulfonyl)-2-oxoindolin-3- \\ ylidene) hydraz-inyl)-4-oxothiazol-5(4H)- ylidene) acetate (11):-}

Equimolar quantities $(0.01 \mathrm{~mol})$ of thiosemicarbazone (10) and dimethylethylenedicarboxylate $(0.01 \mathrm{~mol})$ were dissolved in methanol $(15 \mathrm{~mL})$ and refluxed for $2 \mathrm{~h}$. The solid product formed collected by filtration and washed by hot methanol.

Yield $83.14 \%$ as orange powder from methanol ; mp: $262-264{ }^{\circ} \mathrm{C}$, IR: v/cm ${ }^{-1}: 3190$ ( $\mathrm{NH}), 3042$ (CH-Ar), 2980,2923,2901, 2864, (CH-aliph), 1732, 1701(C=O), $1614(\mathrm{C}=\mathrm{N}) ;{ }^{1} \mathrm{H}$ NMR: $\delta / \mathrm{ppm}=2.93(\mathrm{t}, 4 \mathrm{H}, J=4.2 \mathrm{~Hz}$, $\left.\mathrm{N}\left(\mathrm{CH}_{2}\right)_{2}\right), 3.66$ (t, $\left.4 \mathrm{H}, J=4.1 \mathrm{~Hz}, \mathrm{O}\left(\mathrm{CH}_{2}\right)_{2}\right)$, $3.81\left(\mathrm{~s}, 3 \mathrm{H}, \mathrm{OCH}_{3}\right), 6.77(\mathrm{~s}, 1 \mathrm{H},-\mathrm{CH}=), 7.12$ $(\mathrm{d}, 1 \mathrm{H}, J=8.0 \mathrm{~Hz}, \mathrm{Ar}-\mathrm{H}), 7.75$ (dd, $1 \mathrm{H}, J=8.3$, $1.9 \mathrm{~Hz}, \mathrm{Ar}-\mathrm{H}), 8.1(\mathrm{~s}, 1 \mathrm{H}, \mathrm{Ar}-\mathrm{H}), 11.27,11.64$ (2s, 2H, NH-isatin , NH; exchangeable with $\left.\mathrm{D}_{2} \mathrm{O}\right) ;{ }^{13} \mathrm{C}$ NMR: 46.32, $46.37\left(\mathrm{~N}\left(\mathrm{CH}_{2}\right)_{2}\right), 53.11$ $\left(\mathrm{OCH}_{3}\right), \quad 65.71, \quad 65.75 \quad\left(\mathrm{O}\left(\mathrm{CH}_{2}\right)_{2}\right), \quad 111.60$, $116.18, \quad 120.87, \quad 121.48, \quad 128.06, \quad 133.09$, 142.29, 146.37, 147.89, 158.88, 163.16, 166.35, $168.98(3 \mathrm{C}=\mathrm{O})$; Anal. Calcd for $\mathrm{C}_{18} \mathrm{H}_{17} \mathrm{~N}_{5} \mathrm{O}_{7} \mathrm{~S}_{2}$ (479.48): C, 45.09; H, 3.57; N, 14.61; Found C, 44.97; H, 3.64; N, 14.45\%.

\section{General procedure for preparation of 12a-c,} 13, 14, and 15:-

A mixture of thiosemicarbazone derivatives (10) $(0.01 \mathrm{~mol})$ and phenacyl bromide derivatives, chloroacetylchloride and/ or 2-chloro-Nphenylacetamide $(0.01 \mathrm{~mol})$ and fused sodium acetate $(0.2 \mathrm{~g})$ in methanol $(20 \mathrm{ml})$ was heated under reflux for 4-6 $\mathrm{h}$. The solid product that formed was collected by filtration and washed with hot water.

\section{3-(2-(4-(4-Chlorophenyl)thiazol-2- yl)hydrazono)-5- (morpholinosulfonyl)indolin-2-one (12a):-}

Yield $87.52 \%$ as orange powder from dioxane ; mp: $266-268{ }^{\circ} \mathrm{C}$, IR: $v / \mathrm{cm}^{-1}: 3418$, 3279 ( NH), 3087 (CH-Ar), 2967, 2917, 2858 (CH-aliph), $1698(\mathrm{C}=\mathrm{O}), 1615(\mathrm{C}=\mathrm{N}) ;{ }^{1} \mathrm{H}$ NMR: $\delta / p p m ~ 2.90\left(t, 4 \mathrm{H}, J=4.4 \mathrm{~Hz}, \mathrm{~N}\left(\mathrm{CH}_{2}\right)_{2}\right)$, 3.66 (t, 4H, O $\left.\left(\mathrm{CH}_{2}\right)_{2}\right), 7.15-7.23$ (m, 2H, Ar-H), $7.51(\mathrm{~d}, 1 \mathrm{H}, J=8.0 \mathrm{~Hz}, \operatorname{Ar}-\mathrm{H}), 7.72(\mathrm{~d}, 1 \mathrm{H}, J=$ $8.4 \mathrm{~Hz}, \mathrm{Ar}-\mathrm{H}), 7.76$ (m, 1H, Ar-H), 7.95 (d, 1H, $J=8.0 \mathrm{~Hz}, \mathrm{Ar}-\mathrm{H}), 8.1(\mathrm{~s}, 1 \mathrm{H}, \mathrm{Ar}-\mathrm{H}), 8.55(\mathrm{~s}$, $1 \mathrm{H},-\mathrm{CH}$ thiazol), 11.08, 11.65, (s, 2H, 2NH, exchangeable with $\left.\mathrm{D}_{2} \mathrm{O}\right) ;{ }^{13} \mathrm{C}$ NMR: 46.38 , $46.43\left(\mathrm{~N}\left(\mathrm{CH}_{2}\right)_{2}\right), \quad 65.71, \quad 65.79 \quad\left(\mathrm{O}\left(\mathrm{CH}_{2}\right)_{2}\right)$, $111.82, \quad 120.91, \quad 121.43,127.94,128.55$, $129.23, \quad 130.91,131.30,145.33,146.38$, 153.19, 163.16, $179.27(\mathrm{C}=\mathrm{O})$; Anal. Calcd for $\mathrm{C}_{21} \mathrm{H}_{18} \mathrm{ClN}_{5} \mathrm{O}_{4} \mathrm{~S}_{2}$ (503.98): C, 50.05; H, 3.60; N, 13.90; Found C, 49.82; H, 3.41; N, 14.05\%.

\section{3-(2-(4-(4-Hydroxyphenyl)thiazol-2- yl)hydrazono)-5- (morpholinosulfonyl)indolin-2-one (12b):-}

Yield $88.94 \%$ as red powder from dioxane ; mp: $255-257{ }^{\circ} \mathrm{C}$, IR: $v / \mathrm{cm}^{-1}: 3307,3245$ ( $\mathrm{NH}), 3083$ (CH-Ar), 2978, 2918, 2881 (CHaliph), $1694(\mathrm{C}=\mathrm{O}), 1615(\mathrm{C}=\mathrm{N})$; ${ }^{1} \mathrm{H}$ NMR: $\delta / p p m ~ 2.89\left(\mathrm{t}, 4 \mathrm{H}, J=4.0 \mathrm{~Hz}, \mathrm{~N}\left(\mathrm{CH}_{2}\right)_{2}\right), 3.65$ $\left(\mathrm{t}, 4 \mathrm{H}, J=4.0, \mathrm{O}\left(\mathrm{CH}_{2}\right)_{2}\right), 6.80-6.84(\mathrm{~m}, 2 \mathrm{H}$, Ar-H), 7.03 (s, 1H, Ar-H), 7.15-7.23 (m, 1H, Ar-H), 7.45-7.54 (m, 1H, Ar-H), $7.68(\mathrm{~d}, 1 \mathrm{H}$, $\mathrm{J}=8.0 \mathrm{~Hz}, \operatorname{Ar}-\mathrm{H}), 7.74(\mathrm{~d}, 1 \mathrm{H}, \mathrm{J}=8.0 \mathrm{~Hz}$, Ar-H), $8.54(\mathrm{~s}, 1 \mathrm{H}$, thiazol-H), 9.62, 11.72,13.3 (3s, $3 \mathrm{H}, 2 \mathrm{NH}, \mathrm{OH}$; exchangeable with $\left.\mathrm{D}_{2} \mathrm{O}\right) ;{ }^{13} \mathrm{C}$ NMR: 46.37, $46.45\left(\mathrm{~N}\left(\mathrm{CH}_{2}\right)_{2}\right), \quad 65.71,65.74$ $\left(\mathrm{O}\left(\mathrm{CH}_{2}\right)_{2}\right), 104.9, \quad 112.00, \quad 115.93, \quad 118.99$, $120.91,121.03, \quad 127.66,127.81,128.40$, $128.55, \quad 130.76, \quad 145.17, \quad 151.90, \quad 152.20$, 157.94, 163.7, 172.53; MS:(Mwt.: 485): m/z, 485 [M $\left.\mathrm{M}^{+1},(49.07 \%)\right], 389$ (100\%); Anal. Calcd 
for $\mathrm{C}_{21} \mathrm{H}_{19} \mathrm{~N}_{5} \mathrm{O}_{5} \mathrm{~S}_{2}$ (485.53): C, 51.95; H, 3.94; N, 14.42; Found C, 51.83; H, 3.81; N, 14.54\%.

\section{3-(2-(4-(4-Methoxyphenyl)thiazol-2- yl)hydrazono)-5- (morpholinosulfonyl)indolin-2-one (12c):-}

Yield $85.69 \%$ as orange powder from dioxane ; mp: $264-266{ }^{\circ} \mathrm{C}$, IR: $v / \mathrm{cm}^{-1}: 3250$, 3104 ( 2NH), 3087 (CH-Ar), 2967, 2907, 2858 (CH-aliph), $1691(\mathrm{C}=\mathrm{O}), 1616(\mathrm{C}=\mathrm{N}) ;{ }^{1} \mathrm{H}$ NMR: $\delta / p p m=2.90\left(\mathrm{~s}, 4 \mathrm{H}, \mathrm{N}\left(\mathrm{CH}_{2}\right)_{2}\right), 3.65(\mathrm{~s}$, $\left.4 \mathrm{H}, \mathrm{O}\left(\mathrm{CH}_{2}\right)_{2}\right), 3.89\left(\mathrm{~s}, 3 \mathrm{H}, \mathrm{OCH}_{3}\right), 7.06(\mathrm{~d}, 1 \mathrm{H}$, $J=8.0 \mathrm{~Hz}, \mathrm{Ar}-\mathrm{H}), 7.23(\mathrm{~d}, 1 \mathrm{H}, J=8.4 \mathrm{~Hz}, \mathrm{Ar}-$ $\mathrm{H}), 7.52-7.54(\mathrm{~m}, 1 \mathrm{H}$, Ar-H), 7.66- $7.73(\mathrm{~m}$, $1 \mathrm{H}, \mathrm{Ar}-\mathrm{H}), 7.75-7.77(\mathrm{~m}, 1 \mathrm{H}, \mathrm{Ar}-\mathrm{H}), 7.86(\mathrm{~d}$, $1 \mathrm{H}, J=8.6 \mathrm{~Hz}$, Ar-H), 8.1 (s, $1 \mathrm{H}$, Ar-H), 8.58 (s, 1H, -CH thiazol), 10.97, 11.73, (2s, 2H, $2 \mathrm{NH}$,exchangeable with $\mathrm{D}_{2} \mathrm{O}$ ); Anal. Calcd for $\mathrm{C}_{22} \mathrm{H}_{21} \mathrm{~N}_{5} \mathrm{O}_{5} \mathrm{~S}_{2}$ (499.56): C, 52.89; H, 4.24; N, 14.02; Found C, 52.96; H, 4.36; N, $14.18 \%$.

\section{3-((-4-Iminothiazolidin-2-ylidene) hydrazono)-5-(morpholinosulfonyl) indolin- 2-one (13a):-}

Yield $63.24 \%$ as brown powder from ethanol ; mp: $>300{ }^{\circ} \mathrm{C}$, IR: $v / \mathrm{cm}^{-1}: 3288,3196$ ( 2NH), 3097 (CH-Ar), 2969, 2923, 2857 (CHaliph), $1700(\mathrm{C}=\mathrm{O}), 1613(\mathrm{C}=\mathrm{N}) ;{ }^{1} \mathrm{H}$ NMR: $\delta /$ ppm $2.88\left(\mathrm{t}, 4 \mathrm{H}, J=3.6 \mathrm{~Hz}, \mathrm{~N}\left(\mathrm{CH}_{2}\right)_{2}\right), 3.65$ (s, $\left.4 \mathrm{H}, J=4.0 \mathrm{~Hz}, \mathrm{O}\left(\mathrm{CH}_{2}\right)_{2}\right), 4.02\left(\mathrm{~s}, 2 \mathrm{H}, \mathrm{CH}_{2}\right)$, $7.1(\mathrm{~d}, 1 \mathrm{H}, J=8.0 \mathrm{~Hz}, \mathrm{Ar}-\mathrm{H}), 7.17(\mathrm{~d}, 1 \mathrm{H}, J=$ $8.0 \mathrm{~Hz}, \mathrm{Ar}-\mathrm{H}), 8.11$ (s, 1H, Ar-H), 8.52, 11.22, $11,65(3 \mathrm{~s}, 3 \mathrm{H}, \mathrm{NH}$-isatin, $2 \mathrm{NH}$, exchangeable with $\mathrm{D}_{2} \mathrm{O}$ ); Anal. Calcd for $\mathrm{C}_{15} \mathrm{H}_{16} \mathrm{~N}_{6} \mathrm{O}_{4} \mathrm{~S}_{2}$ (408.45): C, 44.11; H, 3.95; N, 20.58; Found C, 44.02; H, 4.06; N, 20.69\%.

\section{2-((-5-(Morpholinosulfonyl)-2-oxoind-olin-3- ylidene)hydrazono)thiazolidin-5-one (14):-}

Yield $65.21 \%$ as red powder from ethanol ; mp266-268 ${ }^{\circ} \mathrm{C}$, IR: $v / \mathrm{cm}^{-1}: 3284,3194$ ( $2 \mathrm{NH}$ ), 3094, 3051 (CH-Ar), 2978, 2855, 2781 (CHaliph), $1700(\mathrm{C}=\mathrm{O}), 1613(\mathrm{C}=\mathrm{N})$; ${ }^{1} \mathrm{H}$ NMR: $\delta / \mathrm{ppm}=2.88\left(\mathrm{t}, 4 \mathrm{H}, J=3.6 \mathrm{~Hz}, \mathrm{~N}\left(\mathrm{CH}_{2}\right)_{2}\right), 3.65$ $\left(\mathrm{m}, 4 \mathrm{H}, \mathrm{O}\left(\mathrm{CH}_{2}\right)_{2}\right), 4.04\left(\mathrm{~s}, 2 \mathrm{H}, \mathrm{CH}_{2}\right), 7.17(\mathrm{~d}$, $1 \mathrm{H}, J=8.4 \mathrm{~Hz}, \mathrm{Ar}-\mathrm{H}), 7.72(\mathrm{~d}, 1 \mathrm{H}, J=7.2 \mathrm{~Hz}$, Ar-H), 8.10 (s, 1H, Ar-H), 11.23, 11.65 (2s, $2 \mathrm{H}, 2 \mathrm{NH}$, exchangeable with $\mathrm{D}_{2} \mathrm{O}$; MS:(Mwt.:
409): $\mathrm{m} / \mathrm{z}, \quad 409\left[\mathrm{M}^{+1}, \quad(29.65 \%)\right], \quad 402$ (100\%);Anal. Calcd for $\mathrm{C}_{15} \mathrm{H}_{15} \mathrm{~N}_{5} \mathrm{O}_{5} \mathrm{~S}_{2}$ (409.44): C, 44.00; H, 3.69; N, 17.11; Found C, $39.89 ; \mathrm{H}, 3.59 ; \mathrm{N}, 17.42 \%$.

\section{2-oxo-2-(phenylamino)ethyl-2-(5- (morpholinosulfonyl)-2-oxoindolin-3- ylidene)hydrazine-1-carbimidothioate (15):-}

Yield $85.69 \%$ as orange powder from dioxane ; mp: $>300{ }^{\circ} \mathrm{C}$, IR: $v / \mathrm{cm}^{-1}: 3283,3182$ ( 2NH), 3072 (CH-Ar), 2983, 2920, 2859 (CHaliph), $1725(\mathrm{C}=\mathrm{O}), 1614(\mathrm{C}=\mathrm{N})$; ${ }^{1} \mathrm{H}$ NMR: $\delta /$ ppm 2.89 (t, $\left.4 \mathrm{H}, J=4.0 \mathrm{~Hz}, \mathrm{~N}\left(\mathrm{CH}_{2}\right)_{2}\right), 3.65$ (s, $\left.4 \mathrm{H}, J=4.2 \mathrm{~Hz}, \mathrm{O}\left(\mathrm{CH}_{2}\right)_{2}\right), 4.04\left(\mathrm{~s}, 2 \mathrm{H}, \mathrm{CH}_{2}\right)$, 7.08-7.11 (d, 1H, $J=8.0 \mathrm{~Hz}, \operatorname{Ar}-\mathrm{H}), 7.17$ (d, $1 \mathrm{H}, J=8.4 \mathrm{~Hz}, \operatorname{Ar}-\mathrm{H}), 7.42$ (d, $1 \mathrm{H}, J=8.4 \mathrm{~Hz}$, Ar-H), 7.60-7.64 (m, 2H, Ar-H), 7.69- 7.73 (m, 2H, Ar-H), 8.11 (s, 1H, Ar-H), 8.52 (s, 1H, Ar-H), 10.03, 11.23, 11,65, 12.87 (4s, 4H, NHisatin, $\mathrm{NH}$, exchangeable with $\mathrm{D}_{2} \mathrm{O} ;{ }^{13} \mathrm{C}$ NMR: $21.51\left(\mathrm{CH}_{2}\right), 46.37,46.42\left(\mathrm{~N}\left(\mathrm{CH}_{2}\right)_{2}\right), 65.72$, $65.79\left(\mathrm{O}_{(}\left(\mathrm{CH}_{2}\right)_{2}\right), \quad 111.2, \quad 117.67, \quad 120.91$, $121.43, \quad 128.55, \quad 130.92, \quad 131.29, \quad 132.88$, 137.91, 146.38, 148.16, 163.16, 165.30, 174.8, 175.85, 179.26 ; MS: (Mwt.: 502): m/z, 502 [M $\left.\mathrm{M}^{+1},(9.05 \%)\right], 57$ (100\%); Anal. Calcd for $\mathrm{C}_{21} \mathrm{H}_{22} \mathrm{~N}_{6} \mathrm{O}_{5} \mathrm{~S}_{2}$ (502.11): C, 50.19; H, 4.41; N, 16.72; Found C, 50.68; H, 4.31; N, 16.34\%.

\subsection{Antiproliferative activity}

In vitro cytotoxicity of all synthetized compounds were evaluated by using colorimetric assay method (SRB) against three human tumor cell lines including mammary gland breast cancer cell line (MCF-7), human hepatocellular carcinoma cell line (HepG-2), colon carcinoma cell line (HCT-116), they were obtained from VACSERA-Cell Culture Unit, Cairo, Egypt. For comparison, doxorubicin was used as a standard reference drug. RPMI-1640 medium, SRB (SulphoRhodamine-B), DMSO (Dimethyl sulfoxide) and doxorubicin were purchased from (sigma co., St. Louis, USA). Fetal bovine serum was obtained from (GIBCO, UK). The cells were cultured in RPMI-1640 medium with $10 \%$ fetal bovine serum. Antibiotics (penicillin 100 units $/ \mathrm{mL}$ and streptomycin $100 \mu \mathrm{g} / \mathrm{mL}$ ) 
were added at $37{ }^{\circ} \mathrm{C}$ in a $5 \% \mathrm{CO}_{2}$ incubator. The cells were seeded in a 96-well plate at a density of $1.0 \times 104$ cells/well at $37{ }^{\circ} \mathrm{C}$ for $48 \mathrm{~h}$ under $5 \% \mathrm{CO}_{2}$. After incubation, the cells were treated with different concentrations of the tested compounds and incubated for $24 \mathrm{~h}$. Then the medium was discarded. Fixation was carried out by $10 \%$ trichloroacetic acid (TCA) $150 \mu \mathrm{L} /$ well for $1 \mathrm{~h}$ at $4{ }^{\circ} \mathrm{C}$, then wash by water 3 times (TCA reduce SRB protein binding). Wells were stained by SRB $70 \mu \mathrm{L} /$ well for 10 min at room temperature with $0.4 \% 70 \mu \mathrm{L} /$ well (keep in dark place). Then washed with acetic acid $1 \%$ to remove unbound dye (end point: colorless drainage). The plates were subjected to air drying for $24 \mathrm{~h}$. The dye were solubilized with $50 \mu \mathrm{L} /$ well of $10 \mathrm{mMtris}$ base (PH 7.4) for 5 min on a shaker at $1600 \mathrm{rpm}$. The optical density (OD) of each well was measured at 570 nm with an ELISA microplate reader (EXL 800 USA). The inhibitory concentration at $50 \%$ $\left(\mathrm{IC}_{50}\right)$ was determined from the exponential curve of viability versus concentration. The viability was calculated, as (A570 of treated samples/A570 of untreated sample) X 100 and The IC50 values were calculated using the Microsoft Excel. The data were recorded and analyzed to estimate the effects of the tested compounds on cell viability and growth; $\mathrm{IC}_{50}$ values for the tested compounds are reported in Table 1, three independent experiments for each concentration were performed. as previously reported [28].

\section{REFERENCE}

[1] Deng, G.L., Zeng, S., Shen, H., Chemotherapy and target therapy for hepatocellular carcinoma: new advances and challenges. World J. Hepatol. 7, (2015), 787-798.

[2] H. Dua, Y. Huanga, X. Houa, Xi. Quana, J. Jiangb, S. Yuan., Two novel camptothecin derivatives inhibit colorectal cancer proliferation via induction of cell cycle arrest and apoptosis in vitro and in vivo, Eur. J. Pharm. Sci. ,123(2018)546559.

[3] Nguyen M.T., Kumar M. A., Kim J., Lee K.D., Park S., Synthesis, Anticancer and Antioxidant Activity of Novel Carbazole-based Thiazole
Derivatives, Phosphorus Sulfur Silicon Relat. Elem.190 (2015) 191-199.

[4] M. A. Kumar, M. T. Nguyen, K. D. Lee, S. Park, Synthesis and Bioactivity of Novel PhenothiazineBased Thiazole Derivatives, Phosphorus Sulfur Silicon Relat. Elem. 190, (2015)1160-1168.

[5] R. S. Roy, A. M. Gehring, J. C. Milne, P. J. Belshaw, C. T. Walsh, Thiazole and oxazole peptides: biosynthesis and molecular machinery, Nat. Prod. Rep. 16 (1999), 249-263.

[6] K. Taori, V. J. Paul, H. Luesch, Structure and activity of largazole, a potent antiproliferative agent from the Floridian marine cyanobacterium Symploca sp., J. Am. Chem. Soc. 130, (2008), 1806-1807.

[7] Z. Jin, Nat. Prod. Rep., Imidazole, oxazole and thiazole alkaloids, 23, (2006), 464-496.

[8] A. E. Amr, N. M. Sabrry, M. M. Abdalla, B. F. Abdel-Wahab, Synthesis, antiarrhythmic and anticoagulant activities of novel thiazolo derivatives from methyl 2-(thiazol-2ylcarbamoyl) acetate, Eur. J. Med. Chem. 44, (2009),725-735.

[9] T. E. Glotova, M. Y. Dvorko, A. I. Albanov, O. N. Kazheva,G. V. Shilov, and O. A. D'yachenko, 1,3-Dipolar cycloaddition of 3-phenylamino-5phenylimino-1,2,4-dithiazole to 1-acyl-2phenylacetylenes - A new route to functionalized 1,3-thiazole derivatives, Russ. J. Org. Chem. 44, (2008), 1532-1537.

[10] R. K. Yadlapalli, O. P. Chourasia, M. P. Jogi, A. R. Podile, R. S. Perali, Design, synthesis and in vitro antimicrobial activity of novel phenylbenzamido-aminothiazole-based azasterol mimics, Med. Chem. Res., 22(6), (2013), 29752983.

[11] S. Carradoria, D. Rotilia, C. D. Montea, A. Lenoci, A. Mai, Evaluation of a large library of (thiazol-2-yl)hydrazones and analogues as histone acetyltransferase inhibitors: enzyme and cellular studies, Eur. J. Med. Chem.80, (2014),569-578.

[12] B. Parrino, A. Attanzio, V. Spanò, S. Cascioferro, A. Carbone, Synthesis, antitumor activity and CDK1 inhibiton of new thiazole nortopsentin analogues, Eur. J. Med. Chem. 138, (2017), 371383.

[13] W. Xie, Y. Wu , J. Zhang, Q. Mei, Y. Zhang, N. Zhu, R. Liu, H. Zhang,Design, synthesis and biological evaluations of novel pyridone-thiazole hybrid molecules as antitumor agents, Eur. J. Med. Chem.145, (2018), 35-40.

[14] Y.Q. Hu, S. Zhang, Z. Xu, Z.S. Lv, M.L. Liu, L.S. Feng, 4-Quinolone hybrids and their antibacterial 
activities, Eur. J. Med. Chem. 141 (2017) 335345.

[15] M.T. Gabr, N.S. El-Gohary, E.R. Elbendary, M.M. El-Kerdawy, N. Ni, Isatin- $\beta$ thiocarbohydrazones: Microwave-assisted synthesis, antitumor activity and structure-activity relationship, Eur. J. Med. Chem. 128 (2017) 3644.

[16] K.L. Vine, L. Matesic, J.M. Locke, M. Ranson, D. Skropeta, Cytotoxic and anticancer activities of isatin and its derivatives: a comprehensive review from 2000-2008, Anti-cancer Agents in Medicinal Chemistry, 9 (2009) 397-414.

[17] T.L. Devale, J. Parikh, P. Miniyar, P. Sharma, B. Shrivastava, P. Murumlkar, Dihydropyrimidinone-isatin hybrids as novel nonnucleoside HIV-1 reverse transcriptase inhibitors, Bioorg. Chem. 70 (2017) 256-266.

[18] Y.Q. Hu, C. Gao, S. Zhang, L. Xu, Z. Xu, L.S. Feng, X. Wu, F. Zhao, Quinoline hybrids and their antiplasmodial and antimalarial activities, Eur. J. Med. Chem. 139 (2017) 22-47.

[19] Z. Xu, S. Zhang, C. Gao, F. Zhao, Z.S. Lv, L.S. Feng, Isatin hybrids and their anti-tuberculosis activity, Chin. Chem. Lett. 28 (2017) 159-167.

[20] K. Swathi, M. Sarangapani; Synthesis and screening of biologically significant 5-hydroxy isatin derivatives for antioxidant activity, Adv. Exp. Med. Biol., 822 (2015) 129-137.

[21] T. Han, M. Goralski, N. Gaskill, E. Capota, J. Kim, T.C. Ting, Y. Xie, N.S. Williams,

D. Nijhawan, Anticancer sulfonamides target splicing by inducing RBM39 degradation via recruitment to DCAF15, Science 356 (2017), eaal3755.

[22] W.B. Moro, Z. Yang, T.A. Kane, Q. Zhou, S. Harville, C.G. Brouillette, W.J. Brouillette, SAR studies for a new class of antibacterial NAD biosynthesis inhibitors, J. Combin. Chem. 11 (2009) 617-625.

[23] L.S. Simon, F.L. Lanza, P.E. Lipsky, R.C. Hubbard, S. Talwalker, B.D. Schwartz, P.C. Isakson, G.S. Geis, Preliminary study of the safety and efficacy of SC-58635, a novel cyclooxygenase 2 inhibitor: efficacy and safety in two placebocontrolled trials in osteoarthritis and rheumatoid arthritis, and studies of gastrointestinal and platelet effects, Arthritis Rheumatol. 41 (1998)1591-1602.

[24] M. Andrs, J. Korabecny, D. Jun, et al., Phosphatidylinositol 3-kinase (PI3K) and phosphatidylinositol 3-kinase-related kinase
(PIKK) inhibitors: importance of the morpholine ring, J. Med. Chem. 58 (2015) 41-71.

[25] C. Dugave, L. Demange, Cis-trans isomerization of organic molecules and biomolecules: implications and applications, Chem. Rev.103 (2003) 2475-2532.

[26] A. Arrieta, D. Oteagui, A. Zubia, et al., Solventfree thermal and microwave- assisted [3+2] cycloadditions between stabilized azomethine ylides and nitrostyrenes. An experimental and theoretical study, J. Org. Chem. 72 (2007) 43134322 .

[27] A. Polak, Mode of action of morpholine derivatives, Ann. N. Y. Acad. Sci. 554 (1988) 221-228.

[28] Y. A. Ammar, A. M. Sh. El-Sharief, A. Belal , S. Y. Abbas, Y. A. Mohamed, A. B. M. Mehany, A. Ragab, Design, synthesis, antiproliferative activity, molecular docking and cell cycle analysis of some novel (morpholinosulfonyl) isatins with potential EGFR inhibitory activity,Eur. J. Med. Chem.156 (2018), 918-932.

[29] Y. A. Ammar, M. A. M. Sh. El-Sharief, M. M. Ghorab, Y. A. Mohamed, A. Ragab, S. Y. Abbas, New Imidazolidineiminothione, Imidazolidin-2one and Imidazoquinoxaline Derivatives: Synthesis and Evaluation of Antibacterial and Antifungal Activities, Curr, Org. Synth. 13 (2016), 466-475.

[30] M. A. M. Sh El-Sharief, S. Y. Abbas, M. A. Zahran, Y. A. Mohamed, A. Ragab, Y. A. Ammar, New 1,3-diaryl-5-thioxo-imidazolidin2,4-dione derivatives: synthesis, reactions and evaluation of antibacterial and antifungal activities, Z. Naturforsch.B.71 (2016), 875-881.

[31] Y. A. Ammar, S. Y. Abbas, M. A. M. Sh. El-Sharief, M. A. Salem, A. Ragab, Synthesis and characterization of new imidazolidineiminothione and bis-imidazolidineiminothione derivatives as potential antimicrobial agents, Eur. J. Chem. 8 (2017),76-81.

[32] G. A. M. Elhagali, G. A. Elsayed, R. A. Eliswey, A. A. El-Sherif, Molecular modeling and cyclization reactions of 2-(4-oxothiazolidine-2-ylidene) acetonitrile, J. Iran. Chem. Society 15 (2018), 1243-1254.

[33] A. A. Farge, Synthesis and Antimicrobial Activity of 5-(morpholinosulfonyl) isatin Derivatives Incorporating a Thiazole Moiety, Drug Res. 65 (2015), 373-379. 